\title{
Relationship between the electroencephalogram, eye-movements and fluctuations of visual perception
}

BARAY KIRKWOOD

UNIVERSITY OF OTAGO, NEW ZEALAND

EEG activity and eye movements were recorded while Ss inspected a Ganzfeld or diffuse dark figures introduced into a Ganzfeld. Reports of deterioration of visual perception under all stimulus conditions were preceded by a period of reduced eye movement, but were not associated with a retum of EEG alpha activity.

Observers report an experience of blank-out or loss of visual perception after a period of exposure to a uniformly illuminated homogeneous field or Ganzfeld (Cohen, 1957). Moreover, areas of inhomogeneous hue or brightness on an otherwise structureless field seem to fade into the background after a time (Guilford, 1927; Cohen, 1958). A similar adaptational mechanism probably underlies both these effects and the fade-out of stabilized retinal images, but evidence from their physiological correlates is unclear. Cohen \& Cadwallader (1958) reported that susceptibility to Ganzfeld blank-out was positively correlated with the level of alpha activity in Ss' resting EEG. Tepas (1962), however, found that blank-out periods were little related to alpha activity but were strongly associated with periods of reduced eye-movement. This parallels Guilford's (1927) observation that unstabilized images fade when there is little eye movement, but there are no data to hand on the EEG correlates of this effect. Lehmann et al (1957) reported that the fading of stabilized retinal images is associated with the return of EEG alpha trains in some Ss.

The present study investigates the Ganzfeld blankout effect and the fade-out of unstabilized images by simultaneously monitoring both occipital EEG potentials and eye movements using two groups of Ss differentiated on the basis of their levels of resting alpha activity.

Subjects

Six young male Ss having no known visual or neurological defect were used. Four Ss showed frequent alpha trains when resting. Two Ss gave fast, low amplitude EEG traces even when resting with eyes closed.

\section{Apparatus}

EEG, electrooculograph potentials, subject responses and time marks were recorded on a Beckman $\mathrm{RB}$ dynograph. EEG electrodes were attached with Bentonite at the $10-20$ position ipsilateral to the stimulated eye. Eye movement was monitored via $3 \mathrm{~mm}$ disc electrodes placed at the inner and outer canthi of the stimulated eye. Verbal responses from $S$ activated an event marker through a Grason-Stadler voice-operated relay. All recording was carried out with $S$ in an electrically shielded sound-reduced room.

S's non-preferred eye was covered with a patch. The other eye was covered with a cap fashioned from a half table-tennis ball. Four caps were used, one blank to give homogeneous stimulation, the others having patterns described upon their outer surfaces with matt black acrylic paint. Pattern A was a vertical bar $1 \mathrm{~mm} \times 1 \mathrm{~cm}$, Pattern $B$ a vertical $1 \mathrm{~mm}$ bar bisecting the field, and Pattern $\mathrm{C}$ of a lattice of $1 \mathrm{~mm}$ bars spaced $2 \mathrm{~mm}$ between centers. Because the patterns are close to the eye, complete accommodation is impossible and the contours appear diffuse to $\mathrm{S}$. The light source was a 200-W incandescent lamp shining through opal glass diffusing screens to give a luminance of $0.9 \mathrm{ft}$. $-\mathrm{L}$ to the inner surface of a plain cap at $S$ 's position.

\section{Procedure}

S lay supine. Cap A was placed over the eye and $S$ instructed to observe the dark line. All S's spontaneously reported fade-out after a period. They were then instructed to report each further fade-out during the experimental run. The order of viewing each pattern was randomized over Ss. The blank cap was used last, $\mathrm{S}$ being instructed to report if he experienced a loss of vision. At least five reports of fade-out or blank-out were obtained from each $S$ under each stimulus condition.

\section{Results and Discussion}

For all Ss under all stimulus conditions reports of fade-out or blank-out were preceded by a period of at least $3 \mathrm{sec}$. during which eye-movement potentials were negligible. This accords with the findings of Guilford (1927) and Tepas (1962). Mean times taken from the start of each run to report of fade-out or blank-out are shown in Table 1. It can be seen

Table 1. Mean Time (sec) to Fade-out

\begin{tabular}{lcccc} 
Group & Fig. A & Fig. B & Fig. C & Ganzfeld \\
\hline Low $a$ & 4.66 & 7.22 & 21.33 & 41.11 \\
$\mathrm{n}=9$ & $(1.51)$ & $(3.91)$ & $(16.8)$ & $(31.2)$ \\
High $a$ & 5.26 & 6.52 & 15.84 & 22.05 \\
$\mathrm{n}=19$ & $(1.95)$ & $(1.95)$ & $(10.39)$ & $(10.34)$ \\
\hline
\end{tabular}

Note: Standard deviations in brackets below means. 
that adaptation period is positively related to the size and complexity of targets $A, B, C$, but the Ganzfeld data are paradoxical in that the longest adaptation periods are required with this, the least differentiated field. Eye movements while Ganzfeld viewing are, however, more frequent and of greater amplitude than is the case when a patterned field is being inspected. These data support the view that reduced eye movement is a necessary condition for both fade-out and blank-out and that these effects are mediated by processes essentially similar to those involved in the fading of stabilized retinal images.

Data from EEG are, however, more equirocal. Differences in adaptation time between high and low alpha Ss are not statistically significant, although the difference is in the direction predicted by Cohen \& Cadwallader (1958). Visual inspection of the EEG of low alpha Ss shows no discernible change from clear vision through to fade-out. High alpha Ss showed no return of alpha trains either during the fade-out or blank-out phase or in the 3-sec. period immediately preceding loss of vision, in spite of the fact that alpha trains did return intermittently during the longer inspection periods. This contrasts with Lehmann et al (1957) who found a return of alpha accompanying both the fading of stabilized images and the artificial fading out of Images by gross defocussing. But it should be kept in mind that the arousal response involved in merely reporting changes would be expected to cause alpha blocking. Moreover, the blankout experience is emotionally disturbing, and this would also be expected to inhibit alpha activity. Further work with highly practiced high alpha Ss would be necessary to resolve this issue.

\section{References}

Cohen, w. Spatial and textural characteristics of the Ganzfeld. Amer. J. Psychol., 1957, 70, 403-410.

Cohen, W. Color perception in the chromatic Ganzfeld. Amer. J. Psychol., 1958, 71, 390-394.

Guilford, J. P. Fluçtuations of attention with weak visual stimuli. Amer. J. Psychol., 1927, 30, 534-583.

Lehmann, D., Beeler, G. W., \& Fender, D. H. Changes in patterns of the human electroencephalogram during fluctuations of perception of stabilized retinal images. EEG clin. Neurophysiol., $1965,19,336-343$.

Tepas, D. L. The electrophysiological correlates of vision in a uniform field. In M. A. Whitcomb (Ed.), Visual problems in the armed forces. Washington: National Academy of Science, National Research Council, 1962. 